\title{
Energy storage oscillation of metallic glass induced by high-intensity elastic stimulation
}

Cite as: Appl. Phys. Lett. 116, 081901 (2020); https://doi.org/10.1063/1.5140208

Submitted: 25 November 2019 . Accepted: 14 February 2020 . Published Online: 25 February 2020

S. Sohrabi (D), M. X. Li, H. Y. Bai, J. Ma (D), W. H. Wang, and A. L. Greer (D)
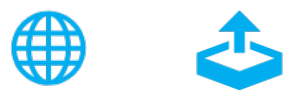

\section{ARTICLES YOU MAY BE INTERESTED IN}

Strain control of phase transition and magnetocaloric effect in $\mathrm{Nd}_{0.5} \mathrm{Sr}_{0.5} \mathrm{MnO}_{3}$ thin films Applied Physics Letters 116, 082402 (2020); https://doi.org/10.1063/1.5134116

A maximum extreme-value distribution model for switching conductance of oxide-RRAM in memory applications

Applied Physics Letters 116, 082901 (2020); https://doi.org/10.1063/1.5141951

High thermoelectric performance of post mechanical treated carbon nanotube films with polystyrene binder

Applied Physics Letters 116, 081902 (2020); https://doi.org/10.1063/1.5135026

Lock-in Amplifiers Find out more today

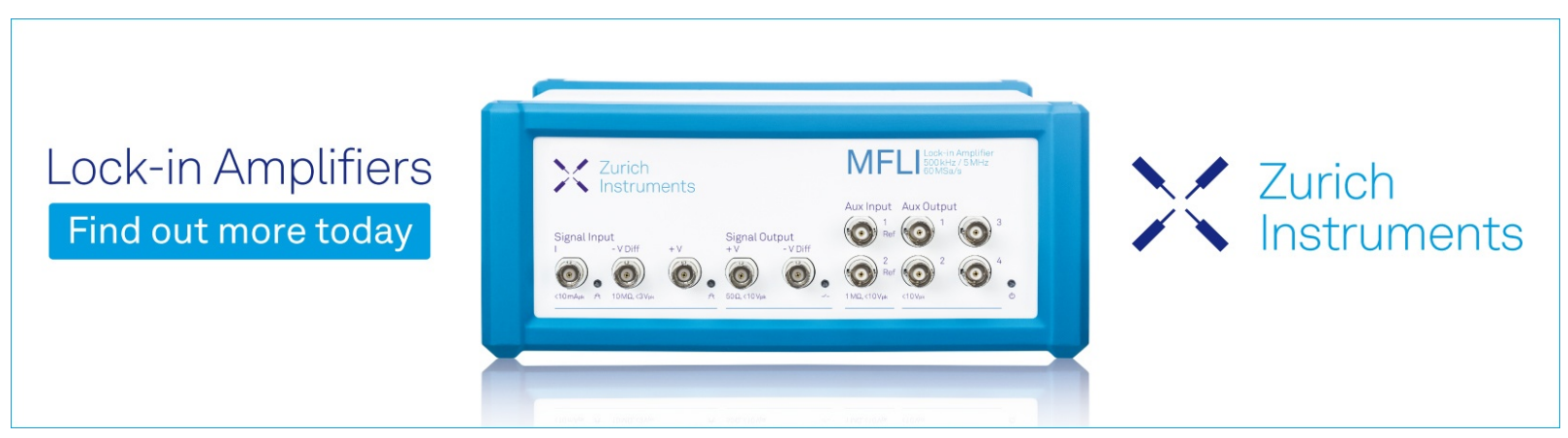




\title{
Energy storage oscillation of metallic glass induced by high-intensity elastic stimulation
}

\author{
Cite as: Appl. Phys. Lett. 116, 081901 (2020); doi: 10.1063/1.5140208 \\ Submitted: 25 November 2019 - Accepted: 14 February 2020 . \\ Published Online: 25 February 2020
}

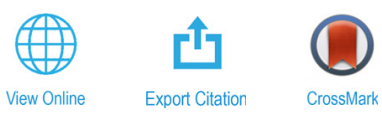

\author{
S. Sohrabi, ${ }^{1,2}$ (D) M. X. Li, ${ }^{1}$ H. Y. Bai, J. Ma, ${ }^{3, a)}$ (D) W. H. Wang, ${ }^{1,2, a)}$ and A. L. Greer $^{4}$ (D)
}

\begin{abstract}
AFFILIATIONS
'Institute of Physics, Chinese Academy of Sciences, Beijing 100190, China

${ }^{2}$ Center of Materials Science and Optoelectronics Engineering, University of Chinese Academy of Sciences, Beijing 100049, China

${ }^{3}$ Guangdong Provincial Key Laboratory of Micro/Nano Optomechatronics Engineering, College of Mechatronics and Control Engineering, Shenzhen University, Shenzhen 518060, People's Republic of China

${ }^{4}$ Department of Materials Science \& Metallurgy, University of Cambridge, 27 Charles Babbage Road, Cambridge CB3 OFS,

United Kingdom
\end{abstract}

${ }^{a)}$ Authors to whom correspondence should be addressed: majiang@szu.edu.cn and whw@iphy.ac.cn

\begin{abstract}
Thermomechanical processing of metallic glasses can, remarkably, induce significant changes in structure and properties, even when the imposed macroscopic strains are well within the elastic regime. The changes can take the glasses to higher-energy "rejuvenated" or lowerenergy "aged" states, with rejuvenation being of particular interest as it improves their mechanical properties. It has generally been assumed that the induced property changes would evolve monotonically with the extent of processing. We show that with sufficiently intense ultrasonic elastic processing, the intrinsic structural competition between damage and repair facilitated by increased atomic mobility can lead to oscillatory energy storage. The uncovering of this behavior forces reconsideration about the range of energy states attainable in metallic glasses by elastic deformation and may provide opportunities.
\end{abstract}

Published under license by AIP Publishing. https://doi.org/10.1063/1.5140208

Metallic glasses (MGs) are out-of-equilibrium systems with the dense packing structure of atoms but no long-range periodicity. MGs are characterized by yield stresses and elastic strain limits far exceeding those of conventional polycrystalline alloys, yet they show poor plasticity in compression due to strain softening and consequent localized flow in shear bands. ${ }^{1}$ For bulk MGs, strain softening is the principal hindrance to their wider application as structural materials. To improve the mechanical properties, many approaches have been adopted, from imposed patterns of residual stress ${ }^{2}$ to component size reduction. ${ }^{3}$

Thermomechanical processing (TMP) is established as a means for improving the mechanical functionality of metallic materials. ${ }^{4}$ When a polycrystalline metal is plastically deformed at room temperature [Fig. 1(a)], part of the mechanical work done is stored ${ }^{5}$ in the material through an increase in the concentration of defects such as dislocations, eventually leading to nanostructured and ultrafine-grained structures with high strength. ${ }^{6}$ Comparably, MGs also can store a fraction of applied cold work, ${ }^{7-9}$ raising their internal energy to resemble states attainable by quenching the initial melt at higher rates. This rejuvenation leads to decreased yield stress and possibly improved plasticity. ${ }^{10}$ Plastic deformation can also induce more relaxed or aged states in MGs. ${ }^{11,12}$ Processing can therefore induce rejuvenation or aging. ${ }^{13}$ More intense processing, a lower processing temperature, and a lower-energy initial state favor rejuvenation. ${ }^{10}$

In contrast to crystalline metals, MGs can even change their structure, gaining or losing energy, as a result of TMP in their nominally elastic regime. ${ }^{14}$ Although the property changes, rejuvenation, ${ }^{15}$ or aging ${ }^{16}$ induced in the elastic regime are usually monotonic, they can be more complex. Elastostatic compression first induces aging and then rejuvenation as the stress is increased, ${ }^{17}$ and under continued thermal cycling, the stored energy increases to a maximum and then decreases. ${ }^{18}$ In this study, we found that the ultrasonic elastic processing can induce an intrinsic structural competition between damage and repair and lead to oscillatory energy storage.

Cylindrical rods of two Zr-based MG compositions $\left(\mathrm{Zr}_{41.2} \mathrm{Ti}_{13.8} \mathrm{Cu}_{12.5} \mathrm{Ni}_{10} \mathrm{Be}_{22.5}\right.$, Vitreloy $\left.1 ; \mathrm{Zr}_{55} \mathrm{Al}_{10} \mathrm{Ni}_{5} \mathrm{Cu}_{30}\right)$ with a diameter of $2 \mathrm{~mm}$ were prepared by copper-mold suction casting. Disks of $1 \mathrm{~mm}$ thickness with flat parallel surfaces were polished from the as-cast rods for the ultrasonic elastic treatment. We applied the relatively intense high-strain-rate elastic deformation offered by ultrasonic hammering (UH). The ultrasonic hammer [see Fig. 1(b)] comprised a 
(a)
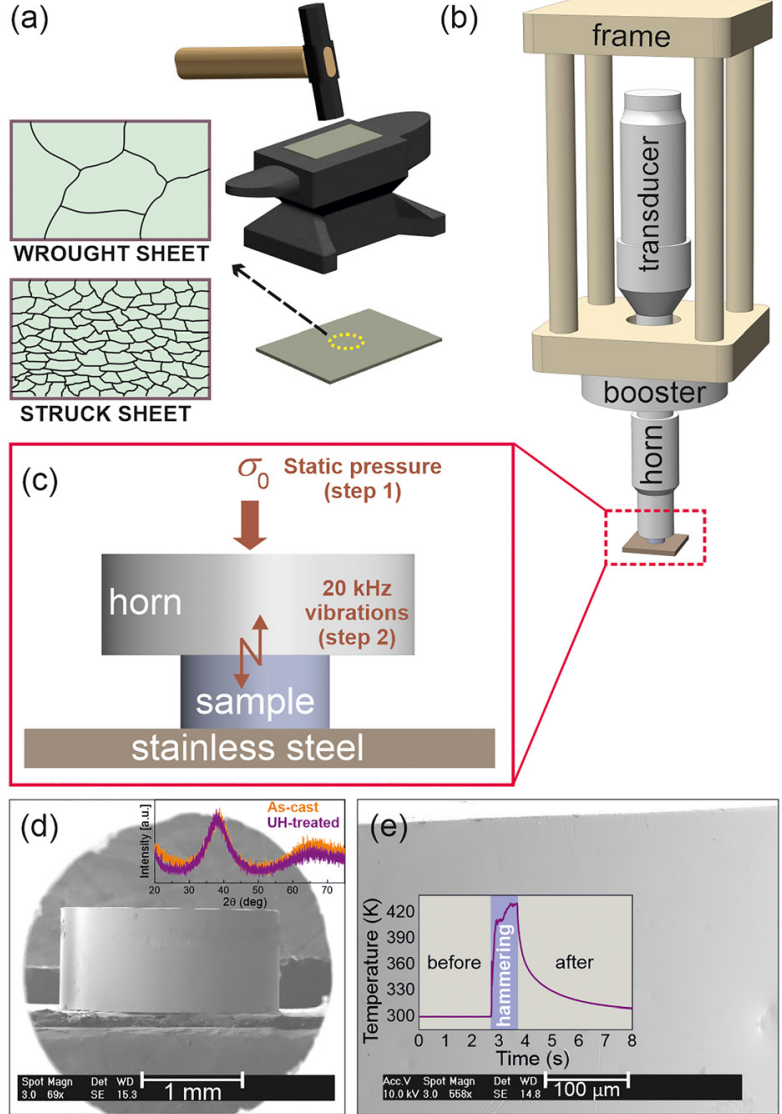

FIG. 1. Hammer processing of metals. (a) When the polycrystalline metallic sheet receives successive impacts of a hammer, a large fraction of the work done is dissipated as sound and heat, and a small fraction $(\sim 5 \%)$ is stored in the material through increasing defect concentrations and refinement of the grains. (b) Schematic illustration of the ultrasonic hammer used in this study. The dashed box is enlarged in (c). (c) Horn-sample configuration during loading. (d) and (e) SEM images of a UH-treated disk of Vit. $1\left(\sigma_{0}=96 \mathrm{MPa}\right.$, hammering time: $1.0 \mathrm{~s}$ ). The inset in (d) shows the XRD patterns from an as-cast sample and the UH-treated disk in (d), which confirms the amorphous nature of the UH-treated surface. The inset in (e) shows the temperature profile of the struck surface.

transducer for converting electrical energy to mechanical vibrations, a booster for amplifying the amplitude of vibrations, and a horn to transfer mechanical vibrations to the workpiece. The disk-shaped specimen was placed in position and then preloaded by slowly lowering the ultrasonic stack, connected by an aluminum frame to the cross-head of the universal testing machine (Zwick Roell), until a chosen value of static pressure $\sigma_{0}$ was reached [step 1 in Fig. 1(c)]. Then, the high-frequency generator operating at a frequency of $20 \mathrm{kHz}$ and a power of $191 \mathrm{~W}$ was turned on, and the ultrasonic vibrations (amplitude of $13 \mu \mathrm{m}$ ) were conveyed by the horn to the specimen for an arbitrary time of $1.0 \mathrm{~s}$ [step 2 in Fig. 1(c)]. Static pressures of 3, 16, 32, 64, or $96 \mathrm{MPa}$ were used for all the MG disks. Additionally, the Vitreloy 1 (Vit. 1) samples were processed with static pressures of 128, 192, 256, $400,800,1440,1920$, or $2880 \mathrm{MPa}$, specifically to study the effect of large pressures; only the two highest static pressures exceeded the yield stress of Vit. $1\left(\sigma_{\mathrm{y}} \approx 1.7 \mathrm{GPa}\right)$.
The amorphicity of the as-cast and UH-processed specimens was characterized by x-ray diffractometry (XRD, a BrukerD8 instrument with $\mathrm{Cu}-K_{\alpha}$ radiation). The morphology of treated specimens was examined by scanning electron microscopy (SEM). A powercompensated Perkin-Elmer DSC 8000 instrument, with a constant flow of high-purity argon, was used to investigate the structural relaxation behavior after the UH process. A heating rate of $0.33 \mathrm{~K} \mathrm{~s}^{-1}$ and a cooling rate of $1.66 \mathrm{~K} \mathrm{~s}^{-1}$ were used in all cases. During each DSC run, the specimen was first heated up to a fully crystallized state and cooled down to room temperature. Then, leaving the specimen in-situ, a second heating scan was performed, the signal from the fully crystallized sample serving as the baseline correction when subtracted from the signal in the first scan. To account for some instrumental drift between scans, the measured signals were adjusted by a linear baseline correction to match the apparent specific heats in the supercooled liquid regions, as applied elsewhere.

The hardness measurements were carried out using a Vickers microhardness tester. Furthermore, cubic specimens of annealed Vit. 1 MG were treated by the UH process and then characterized by resonant ultrasound spectroscopy (RUS) and compression tests (see the supplementary material).

With static pressures in the ranges used $\left(\sigma_{0}=3-96 \mathrm{MPa}\right)$, the treated samples show no evidence of plastic deformation. Figures $1(\mathrm{~d})$ and 1 (e) show a disk of the commercial MG Vit. 1 after UH for $1.0 \mathrm{~s}$ at $\sigma_{0}=96 \mathrm{MPa}$ as a representative sample. The XRD pattern [inset in Fig. 1(d)] confirms that the struck surface of UH-treated samples remains amorphous. The temperature history [see the inset in Fig. 1(e)] shows that there is local heating, and the temperature of the struck surface increases up to $430 \mathrm{~K}$. From this temperature profile, an atomic diffusion distance can be estimated, ${ }^{20}$ which is so small that any annealing effects of the temperature rise can be negligible. Furthermore, increasing $\sigma_{0}$ even to $1.44 \mathrm{GPa}$ (corresponding to $\sim 85 \%$ of the yield stress) leaves Vit. 1 samples undeformed, but larger $\sigma_{0}$ indeed ultimately fractures the sample and leads to remarkable shear banding throughout the disk (see Fig. S1, supplementary material).

The effects of UH on the as-cast specimens were monitored using DSC. Figure 2(a) shows the broad spectra of exothermic relaxations preceding glass transition temperature $T_{\mathrm{g}}$ for the as-cast specimens (DSC traces for the whole temperature range are shown in Fig. S2). The enthalpy of relaxation $\Delta H_{\text {rel }}$ can be calculated by integrating the heat release from the onset of relaxation $\left(T_{\text {onset }}\right)$ to the end of the relaxation spectrum (near to $T_{\mathrm{g}}$ ). $\Delta H_{\text {rel }}$ is the excess enthalpy of the glass over its relaxed state and can be used to quantify the extent of rejuvenation or aging induced by different treatments. ${ }^{7,10,14}$ For each set of $\mathrm{UH}$ conditions, three to five samples were processed and then used for DSC; in this way, the reproducibility of the results could be assessed, and a standard deviation was derived for the value of $\Delta H_{\text {rel }}$.

For a UH processing time of $1.0 \mathrm{~s}$, the effects of static pressure are diverse [Fig. 2(b)]. In $\mathrm{Zr}_{55} \mathrm{Al}_{10} \mathrm{Ni}_{5} \mathrm{Cu}_{30} \mathrm{MG}, \Delta H_{\text {rel }}$ rises at $\sigma_{0}=3 \mathrm{MPa}$ by as much as $\sim 60 \%$ compared to the value in the as-cast glass (an increase comparable to rejuvenation by room-temperature elastostatic loading ${ }^{21}$ and cryogenic thermal cycling ${ }^{18}$ ) and decreases by as much as $\sim 27 \%$ (aging) at $\sigma_{0}=64 \mathrm{MPa}$, ultimately tending to an enthalpy similar to that of the as-cast state. The Vit. 1 MG shows an initial sharp increase in $\Delta H_{\text {rel }}$ followed by oscillations between states variously rejuvenated with respect to the as-cast glass. This non-monotonic trend persists even on increasing to $\sigma_{0}=400 \mathrm{MPa}$ 

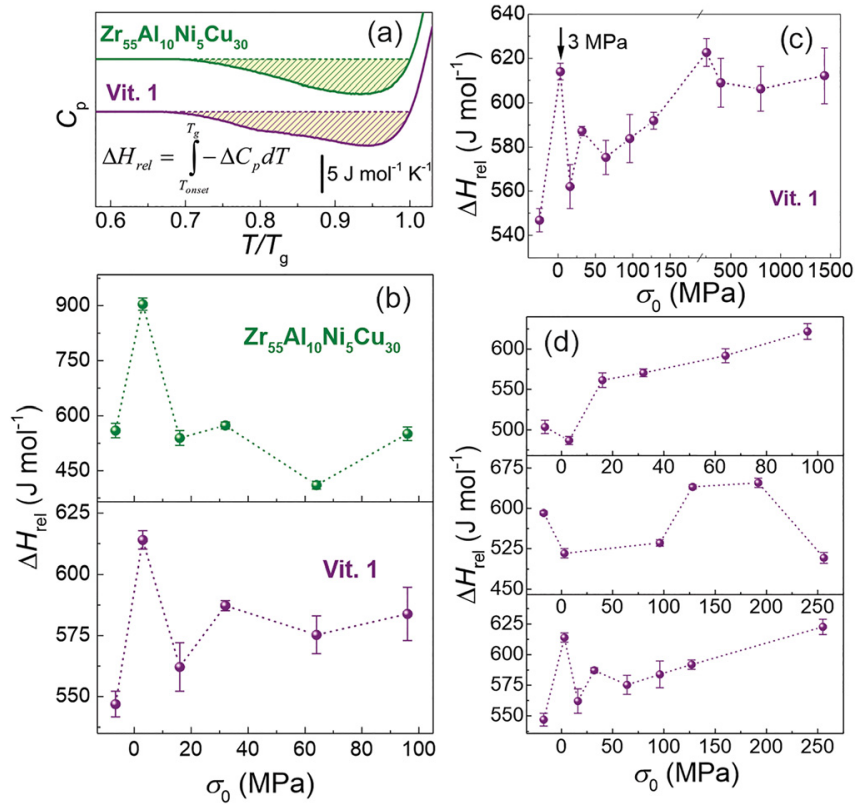

FIG. 2. Stored energy induced by UH. (a) Integrating the specific heat capacity $C_{p}$ (shaded areas) driven from DSC curves (Fig. S2) gives the enthalpy of relaxation $\Delta H_{\text {rel }}$. (b) The effect of static stress $\sigma_{0}$ on the changes in $\Delta H_{\text {rel }}$ induced by UH in different alloys. (c) The effect of extending the static stress $\left(\sigma_{0}\right)$ in Vit. 1. (d) The effect of $\sigma_{0}$ on $\Delta H_{\text {rel }}$ changes in three rods of Vit. 1 with different initial enthalpies, showing the importance of the thermal history of the sample. In (b)-(d), the data points preceding $\sigma_{0}=0 \mathrm{MPa}$ refer to $\Delta H_{\text {rel }}$ for the as-cast conditions

[Fig. 2(c)], and under larger pressures, $\Delta H_{\text {rel }}$ holds its value, attributable to overloading and negligible hammering effects. ${ }^{22}$ The $\Delta H_{\text {rel }}$ data in Figs. 2(b) and 2(c) suggest that the structural evolution induced by $\mathrm{UH}$ is generally non-monotonic.

The effects of $\mathrm{UH}$ are compared for three rods of Vit. 1 cast at different cooling rates [Fig. 2(d)]. The variation of $\Delta H_{\text {rel }}$ as a function of $\sigma_{0}$ in each case is non-monotonic but correlated with the starting condition. The slowest cooled (most relaxed; $\Delta H_{\text {rel }}=504 \mathrm{~J} \mathrm{~mol}^{-1}$ ) glass shows the greatest rejuvenation on $\mathrm{UH}$ treatment. The fastest cooled glass $\left(\Delta H_{\text {rel }}=591 \mathrm{~J} \mathrm{~mol}^{-1}\right)$ ultimately shows aging. The intermediate glass $\left(\Delta H_{\text {rel }}=547 \mathrm{~J} \mathrm{~mol}^{-1}\right)$ shows moderate rejuvenation. Thus, as expected, ${ }^{10}$ the direction of the structural change (aging or rejuvenation) is affected not only by the conditions under which the glass is treated but also by its initial condition: a relaxed initial glass is more likely to be rejuvenated and vice versa.

We test whether non-monotonic behavior (Fig. 2) is systematic by correlating $\Delta H_{\text {rel }}$ with other properties. Prior to the DSC runs [Fig. 2(b)] on $\mathrm{Zr}_{55} \mathrm{Al}_{10} \mathrm{Ni}_{5} \mathrm{Cu}_{30}$ and Vit. 1 glasses, the Vickers microhardness of the disks was measured on the UH-struck surface. For this glass, higher $\mathrm{HV}$ is correlated with lower $\Delta H_{\text {rel }}$ [see Fig. 3(a)], as expected for a denser, more relaxed glass. Furthermore, the hardness of the $\mathrm{Zr}_{55} \mathrm{Al}_{10} \mathrm{Ni}_{5} \mathrm{Cu}_{30} \mathrm{MG}$ disk treated at the static stress of $3 \mathrm{MPa}$ was measured on the opposite surface (i.e., opposite to the struck surface) and along the cross section of the disk [Fig. 3(b)]. It can be observed that the hardness values on these surfaces are very close to the hardness value of the UH-struck surface (HV 480), implying that UH has influenced the whole of the sample homogeneously.
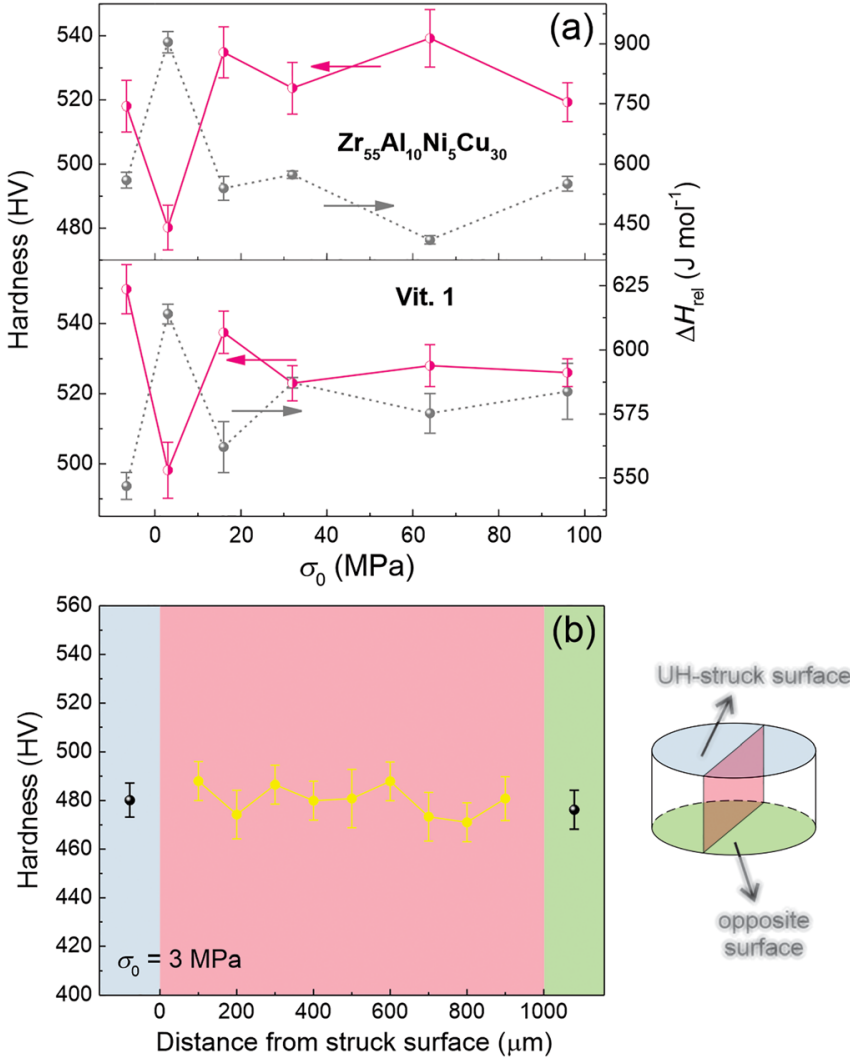

FIG. 3. (a) The effect of $\sigma_{0}$ on hardness and $\Delta H_{\text {rel }}$ in $\mathrm{Zr}_{55} \mathrm{Al}_{10} \mathrm{Ni}_{5} \mathrm{Cu}_{30}$ and Vit. 1 samples. (b) The values of hardness on the struck surface, the opposite surface, and along the cross section of the $\mathrm{Zr}_{55} \mathrm{Al}_{10} \mathrm{Ni}_{5} \mathrm{Cu}_{30}$ sample treated at the static stress of $3 \mathrm{MPa}$.

Further correlations were explored for Vit. $1 \mathrm{MG}$. In annealed samples, UH leads to oscillations in shear modulus as a function of $\sigma_{0}$ at a given hammering time [Fig. 4(a)]. When the UH process intensifies, the treated samples show compressive plasticity sometimes lower, sometimes higher than that of the as-annealed glass [Figs. 4(b) and 4(c)]. Figure 4(d) shows that the higher modulus is well correlated with lower plasticity (as expected for a denser, more relaxed glass). These correlated property changes force the conclusion that there is oscillatory energy storage as a function of the extent of UH.

Even simple thermal annealing of melt-spun MG ribbons appears capable of giving oscillatory changes in properties, ${ }^{23}$ but these effects are complicated by possible evolving structural anisotropy (and anisotropic dimensional changes) in the samples. The broad distribution of relaxation times in MGs can lead to non-monotonic (but not oscillatory) property changes after two-stage annealing. ${ }^{24,25}$ However, the TMP of MGs has been expected to give monotonic changes.

The oscillatory response of glasses is frequently seen in viscous flow of the supercooled liquid state above $T_{\mathrm{g}}$. At fixed temperature and imposed strain rate, the stress rises and shows a maximum as viscous flow starts. At higher strain rates, this stress overshoot can be followed not by monotonic decay to the steady-state flow stress but rather by stress oscillations. This effect, corresponding to oscillatory 

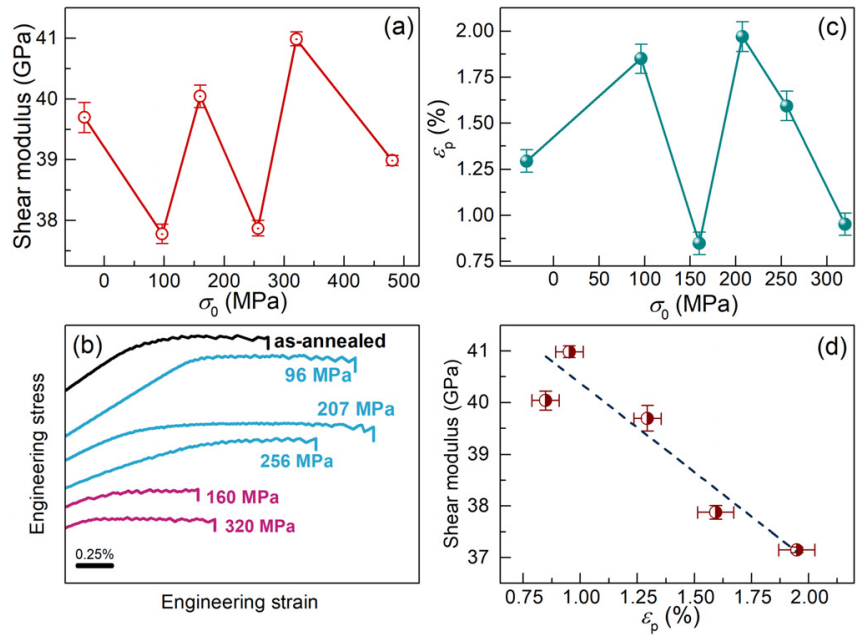

FIG. 4. Oscillatory changes in mechanical properties. (a) Variation of shear modulus with $\sigma_{0}$ in annealed (partially relaxed) Vit. 1 glass (hammering time: $1.0 \mathrm{~s}$ ). (b) Compressive stress-strain curves for partially relaxed Vit. 1 glass hammered at different values of $\sigma_{0}$. (c) The variation of plasticity $\left(\varepsilon_{p}\right)$ with the extent of UH. (d) The correlation between plasticity $\left(\varepsilon_{\mathrm{p}}\right)$ and shear modulus. In (a) and (c), the data points preceding $\sigma_{0}=0 \mathrm{MPa}$ refer to shear modulus in the as-annealed sample. The error bars indicate the root mean square errors from measurements on three annealed samples treated under the same UH conditions.

relaxation of the viscosity, was first seen for glassy polymers, ${ }^{26}$ then for MGs, ${ }^{27}$ and for a borosilicate glass. ${ }^{28}$

The observed oscillations in MG flow stress have been modeled in terms of changes in the structural relaxation time $\tau$ in the supercooled liquid. ${ }^{27}$ During flow at a constant strain rate, from maximum to minimum flow stress corresponds to a decrease in $\tau$ by a factor of $\sim 5,{ }^{27}$ i.e., the relative rejuvenation that would be achieved by forming the glass on cooling five times faster. In present work, the extent of the $\Delta H_{\text {rel }}$ variation induced by $\mathrm{UH}$ varies widely, but it is as high as $500 \mathrm{~J}$ $\mathrm{mol}^{-1}$ for $\mathrm{Zr}_{55} \mathrm{Al}_{10} \mathrm{Ni}_{5} \mathrm{Cu}_{30} \mathrm{MG}$. This is $\sim 5 \%$ of the heat of melting and would correspond to the degree of rejuvenation (or aging) associated with a factor of 100 change in the cooling rate at which the glass was formed. ${ }^{10}$ Therefore, the amplitude of oscillations realized in a MG in the elastic regime can exceed that seen in viscous flow. ${ }^{27}$

The flow behavior of materials under oscillatory excitations can be delineated by the Pipkin diagram. ${ }^{29}$ Figure 5 shows the Pipkin map for the flow behavior of MGs excited by various routes of oscillatory loading. The diagram illustrates strain amplitude as a function of the Deborah number $(\mathrm{De})$. De is the ratio of the relaxation time to the observation time, ${ }^{30}$ and for oscillatory loading conditions, De can be expressed as ${ }^{31}$

$$
D e=\tau_{\mathrm{r}} \omega,
$$

where $\tau_{\mathrm{r}}$ is the material relaxation time and $\omega$ is the frequency of oscillation. When $D e \ll 1$, the material has a liquid-like (Newtonian) flow behavior, and when $D e \gg 1$, the material has a purely elastic behavior. In the moderate values of $D e$, the rheological nature of material's flow is linear/nonlinear viscoelasticity depending on the amplitude of oscillations.

During the low amplitude oscillatory excitation of MGs such as dynamic mechanical spectroscopy (DMS), ${ }^{32}$ the common strain

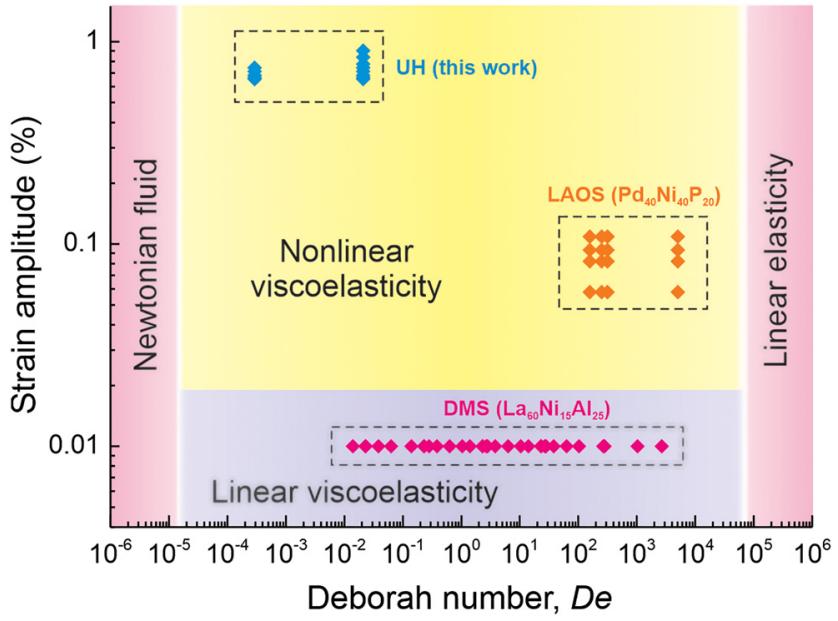

FIG. 5. Pipkin diagram illustrating the flow behavior of MGs under different oscillatory excitation protocols. The diagram is useful to predict viscoelastic (linear/nonlinear) behavior of materials at different values of strain amplitude and De. The lines of demarcation between different flow regimes are schematic. For details about the estimation of De and strain amplitudes, see Table S(a) and supplementary material.

amplitudes used are quite small (typically $\sim 0.01 \%$ ), ${ }^{33}$ and MGs show linear viscoelastic behavior ( $D e$ for $\mathrm{La}_{60} \mathrm{Ni}_{15} \mathrm{Al}_{25} \mathrm{MG}$ is between $\sim 10^{-2}$ and $\sim 10^{4}$ ). However, when a high field excitation is applied, as in large amplitude oscillatory spectroscopy (LAOS) ${ }^{34}$ of $\mathrm{Pd}_{40} \mathrm{Ni}_{40} \mathrm{P}_{20}$ MG where $D e>1$, the viscoelastic response would be nonlinear, and the deviation from linear elasticity (Hooke's law) becomes evident. In our UH experiments, the strain amplitudes range from $\sim 0.65 \%$ to $\sim 0.9 \%$ (see the supplementary material for strain considerations), and these values are quite larger than the common strain amplitudes set during DMS and LAOS excitations. Furthermore, De values for UH excitation of Vit. 1 and $\mathrm{Zr}_{55} \mathrm{Al}_{10} \mathrm{Ni}_{5} \mathrm{Cu}_{30}$ MGs are estimated to be lower than unity. It has been proposed that MGs show serrated (nonlinear) flow behavior when $D e<1 .^{35}$ Therefore, UH excitation lies in the nonlinear viscoelasticity regime of the Pipkin map where nonlinear responses such as oscillatory or serrated behavior are expected.

In summary, the high-intensity processing of MGs within the elastic regime could induce both rejuvenation and aging during the same treatment in a pattern of energy storage oscillations. Consistent with the structural oscillations, mechanical properties also show oscillatory changes. The findings stress the importance of considering possible effects of energy storage oscillations in optimizing the TMP of MGs to broaden the range of available energy states and have significance for the interpretation and tuning of property changes in MGs.

See the supplementary material for the detailed description on the sample preparation, characterization procedures, and estimations of strain amplitude and Deborah number.

We thank P. Luo and C. Yang for experimental assistance and P. Wen and Y. H. Sun for helpful discussions. This work was supported by the NSF of China (Nos. 11790291 and 61888102), the Strategic Priority Research Program of the Chinese Academy of Sciences (No. XDB30000000), and the Key Research Program of 
Frontier Sciences (No. QYZDY-SSW-JSC017). J.M. acknowledges the financial support from the Natural Science Foundation of Guangdong Province (Grant No. 2019B030302010) and the NSF of China (Grant No. 51871157); S.S. from the CAS-TWAS President's Ph.D. Fellowship Program; and A.L.G. from the European Research Council (No. ERC-2015-AdG-695487: ExtendGlass).

\section{REFERENCES}

${ }^{1}$ C. A. Schuh, T. C. Hufnagel, and U. Ramamurty, "Mechanical behavior of amorphous alloys," Acta Mater. 55(12), 4067-4109 (2007).

${ }^{2}$ S. Scudino, B. Jerliu, S. Pauly, K. B. Surreddi, U. Kühn, and J. Eckert, "Ductile bulk metallic glasses produced through designed heterogeneities," Scr. Mater. 65(9), 815-818 (2011).

${ }^{3}$ D. Z. Chen, X. W. Gu, Q. An, W. A. Goddard, and J. R. Greer, "Ductility and work hardening in nano-sized metallic glasses," Appl. Phys. Lett. 106(6), 061903 (2015).

${ }^{4} \mathrm{R}$. Valiev, "Nanostructuring of metals by severe plastic deformation for advanced properties," Nat. Mater. 3(8), 511-516 (2004).

${ }^{5}$ M. B. Bever, D. L. Holt, and A. L. Titchener, "The stored energy of cold work," Prog. Mater. Sci. 17, 5-177 (1973).

${ }^{6} \mathrm{Y}$. Wang, M. Chen, F. Zhou, and E. Ma, "High tensile ductility in a nanostructured metal," Nature 419(6910), 912-915 (2002).

${ }^{7}$ H. S. Chen, "Stored energy in a cold-rolled metallic glass," Appl. Phys. Lett. 29(6), 328-330 (1976).

${ }^{8}$ F. Q. Meng, K. Tsuchiya, S. Ii, and Y. Yokoyama, "Reversible transition of deformation mode by structural rejuvenation and relaxation in bulk metallic glass," Appl. Phys. Lett. 101(12), 121914 (2012).

${ }^{9}$ J. Pan, Y. X. Wang, Q. Guo, D. Zhang, A. L. Greer, and Y. Li, "Extreme rejuvenation and softening in a bulk metallic glass," Nat. Commun. 9(1), 560 (2018).

${ }^{10} \mathrm{Y}$. Sun, A. Concustell, and A. L. Greer, "Thermomechanical processing of metallic glasses: Extending the range of the glassy state," Nat. Rev. Mater. 1, 16039 (2016).

${ }^{11}$ F. O. Méar, B. Lenk, Y. Zhang, and A. L. Greer, "Structural relaxation in a heavily cold-worked metallic glass,” Scr. Mater. 59(12), 1243-1246 (2008).

${ }^{12}$ Z. T. Wang, J. Pan, Y. Li, and C. A. Schuh, "Densification and strain hardening of a metallic glass under tension at room temperature," Phys. Rev. Lett. 111(13), 135504 (2013).

${ }^{13}$ D. J. Lacks and M. J. Osborne, "Energy landscape picture of overaging and rejuvenation in a sheared glass," Phys. Rev. Lett. 93(25), 255501 (2004).

${ }^{14}$ A. L. Greer and Y. H. Sun, "Stored energy in metallic glasses due to strains within the elastic limit," Philos. Mag. 96(16), 1643-1663 (2016).

${ }^{15}$ S.-C. Lee, C.-M. Lee, J.-C. Lee, H.-J. Kim, Y. Shibutani, E. Fleury, and M. L. Falk, "Structural disordering process of an amorphous alloy driven by the elastostatic compression at room temperature," Appl. Phys. Lett. 92(15), 151906 (2008).

${ }^{16}$ Y. M. Wang, M. Zhang, and L. Liu, "Mechanical annealing in the homogeneous deformation of bulk metallic glass under elastostatic compression," Scr. Mater. 102, 67-70 (2015)
${ }^{17}$ M. Zhang, Y. M. Wang, F. X. Li, S. Q. Jiang, M. Z. Li, and L. Liu, "Mechanical relaxation-to-rejuvenation transition in a Zr-based bulk metallic glass," Sci. Rep. 7(1), 625 (2017).

${ }^{18}$ S. V. Ketov, Y. H. Sun, S. Nachum, Z. Lu, A. Checchi, A. R. Beraldin, H. Y. Bai, W. H. Wang, D. V. Louzguine-Luzgin, M. A. Carpenter, and A. L. Greer, "Rejuvenation of metallic glasses by non-affine thermal strain," Nature 524(7564), 200-203 (2015).

${ }^{19}$ M. Stolpe, J. J. Kruzic, and R. Busch, "Evolution of shear bands, free volume and hardness during cold rolling of a Zr-based bulk metallic glass," Acta Mater. 64, 231 (2014).

${ }^{20}$ F. Faupel, W. Frank, M.-P. Macht, H. Mehrer, V. Naundorf, K. Rätzke, H. R. Schober, S. K. Sharma, and H. Teichler, "Diffusion in metallic glasses and supercooled melts," Rev. Mod. Phys. 75(1), 237-280 (2003).

${ }^{21}$ K.-W. Park, C.-M. Lee, M. Wakeda, Y. Shibutani, M. L. Falk, and J.-C. Lee, "Elastostatically induced structural disordering in amorphous alloys," Acta Mater. 56(19), 5440-5450 (2008).

${ }^{22} \mathrm{C}$. J. Nonhof and G. A. Luiten, "Estimates for process conditions during the ultrasonic welding of thermoplastics," Polym. Eng. Sci. 36(9), 1177-1183 (1996).

${ }^{23}$ A. L. Greer and J. A. Leake, "Structural relaxation and crossover effect in a metallic glass," J. Non-Cryst. Solids, J. Non. Cryst. Solids 33(2), 291 (1979).

${ }^{24} \mathrm{~L}$. Hu and Y. Yue, "Secondary relaxation in metallic glass formers: Its correlation with the genuine Johari-Goldstein relaxation," J. Phys. Chem. C 113(33), 15001 (2009).

${ }^{25}$ J. Moya, F. Audebert, and H. Sirkin, "Structural relaxation on $\mathrm{Fe}_{44} \mathrm{Ni}_{38} \mathrm{~B}_{18}$ amorphous alloy by Young's modulus measurements," Scr. Metall. Mater. 27(8), 1045 (1992).

${ }^{26}$ J. D. Huppler, I. F. Macdonald, E. Ashare, T. W. Spriggs, R. B. Bird, and L. A. Holmes, "Rheological properties of three solutions. Part ii. Relaxation and growth of shear and normal stresses," Trans. Soc. Rheol. 11(2), 181-204 (1967).

${ }^{27} \mathrm{H}$. Kato, Y. Kawamura, A. Inoue, and H.-S. Chen, "Transition from linear to nonlinear viscoelasticity during deformation in a Zr-based glassy alloy," Mater. Trans., JIM 41(9), 1202-1207 (2000).

${ }^{28}$ W. D. Liu, L. C. Zhang, and K. Mylvaganam, "Relaxation oscillation of borosilicate glasses in supercooled liquid region," Sci. Rep. 7(1), 15872 (2017).

${ }^{29}$ A. C. Pipkin, Lectures on Viscoelasticity Theory, Applied Mathematical Sciences Vol. 7, edited by J. P. LaSalle, F. John, and L. Sirovich (SpringerVerlag, New York, 1972), Chap. 8.

${ }^{30}$ M. Reiner, “The Deborah number," Phys. Today 17(1), 62 (1964).

${ }^{31}$ A. S. Khair, "Large amplitude oscillatory shear of the Giesekus model," J. Rheol. 60(2), 257 (2016)

${ }^{32}$ C. Liu, E. Pineda, and D. Crespo, "Mechanical relaxation of metallic glasses: An overview of experimental data and theoretical models," Metals 5(2), 1073 (2015).

${ }^{33}$ J. C. Qiao, Y.-J. Wang, J. M. Pelletier, L. M. Keer, M. E. Fine, and Y. Yao, "Characteristics of stress relaxation kinetics of La60Ni15Al25 bulk metallic glass," Acta Mater. 98, 43 (2015).

${ }^{34}$ B. Riechers and K. Samwer, "Nonlinear response and avalanche behavior in metallic glasses," Eur. Phys. J. Spec. Top. 226(14), 2997 (2017).

${ }^{35}$ M. Q. Jiang, S. Y. Jiang, Z. Ling, and L. H. Dai, "Smaller Deborah number inducing more serrated plastic flow of metallic glass," Comput. Mater. Sci. 46(3), 767 (2009). 